Research Article

\title{
Numerical Simulation of Gravity Anomaly Based on the Unstructured Element Grid and Finite Element Method
}

\author{
Chenyang $X u \mathbb{D}^{1,2}$ and Zhijun Huo ${ }^{1,2}$ \\ ${ }^{1}$ College of Geo-Exploration Science and Technology, Jilin University, Changchun 130026, China \\ ${ }^{2}$ Ministry of Land and Resources Key Laboratory of Applied Geophysics, Jilin University, Changchun 130026, China
}

Correspondence should be addressed to Chenyang Xu; cyxu15@mails.jlu.edu.cn

Received 4 July 2020; Accepted 18 August 2020; Published 29 September 2020

Academic Editor: Gaetano Giunta

Copyright (c) 2020 Chenyang Xu and Zhijun Huo. This is an open access article distributed under the Creative Commons Attribution License, which permits unrestricted use, distribution, and reproduction in any medium, provided the original work is properly cited.

\begin{abstract}
Finite element method is an important method to solve mathematical problems in engineering. Many mathematical equations are difficult to solve, but it becomes very simple after using the finite element method. In this paper, the finite element method is applied to the calculation of gravity anomaly. First, the variational equation of gravity anomaly calculation is established, and then the gravity anomaly value ten times the distance away from the anomaly body is used as the boundary condition. By comparing the gravity anomaly obtained by solving the stiffness matrix with the analytical solution, it can be found that the method in this paper has high accuracy. Finally, the model of Jinchuan copper nickel deposit is used for calculation, and the calculated gravity anomaly field is inverted with Growth3D. It can be found that the inversion result is very close to the model, which verifies the effectiveness of the algorithm in this paper.
\end{abstract}

\section{Introduction}

Finite element method is an effective method to solve mathematical problems [1]. It is based on the variational principle and the weighted residual method. Its basic solution idea is to divide the calculation domain into finite nonoverlapping elements [2]. In each element, select some suitable nodes as the interpolation points of the solution function and rewrite the variables in the differential equation into a linear expression composed of the node values of each variable or its derivative and the selected interpolation function. By means of variational principle or weighted residual method, the differential equation is solved discretely [3]. Different weight functions and interpolation functions are used to form different finite element methods. The finite element method was first used in structural mechanics, and then it was used in the numerical simulation of hydrodynamics with the development of computers [4].

The model built by the rectangular or structural grid has the advantages of convenient calculation. It can be calculated by finite difference, finite element, finite volume, and other algorithms. It has been favored by many experts and scholars [5]. However, the regular body grid also has great limitations. When the structure of the model body is relatively complex, the grid often has no advantages and there is no way to carry out accurate modeling, so that the accuracy of the subsequent simulation results is not reliable [6]. In order to overcome this problem, it can be considered to increase the grid density to capture the accuracy of numerical simulation solution, but it will greatly increase the amount of calculation [7]. Based on the above considerations, the use of unstructured grid modeling is undoubtedly a very effective method. This method can encrypt the grid in the important area and sparse the grid in the background area to save the memory of the computer. At the same time, the accurate description of the model also provides a strong support for the accuracy of numerical simulation [8]. At the same time, using the finite element method for numerical simulation will also achieve good results [9].

In the field of Engineering Geophysics, inversion imaging of the underground target is one of the most important targets [10]. In the process of inversion, the forward 
algorithm is one of the most important components. The accuracy of forward simulation and the establishment of the forward model are the key to the success of forward calculation [11]. The finite element method is very powerful in the calculation of a complex model, and the Delaunay grid has good adaptability in the establishment of the complex model [12-14]. The combination of the two will make the calculation of gravity anomaly more advantageous. When the work of forward modeling is completed, the inversion of geophysical methods will progress together [15].

In the inversion process of geophysics, the most commonly used methods are the least-square method and the minimum structure method. At the same time, these two methods also perform well in the calculation of potential field. However, in the inversion process, the underground geological body is often divided into a combination of finite elements, so it is also a meaningful work to refine the internal area of the geological body locally [16].

With the development of the computer technology, the finite element method is more and more widely used to solve the boundary value problems of the Poisson equation in various mathematical technical problems [17]. The calculation of the gravity anomaly generated by the three-dimensional geological body can be transformed into the boundary value problem of the Poisson equation, so the finite element method can be used to solve the gravity anomaly field of the three-dimensional geological model, and it also provides an effective new method for the calculation of the gravity anomaly generated by the abnormal body [18].

In this paper, the unstructured grid and finite element method are used for numerical calculation of gravity anomalies, and synthetic data are used for analysis and evaluation of the results of this paper. Finally, the actual model of Jinchuan copper nickel deposit is modeled by the unstructured grid, and then the finite element method is used for numerical simulation, and the results of simulation are inverted by the growth code, which shows that the inverse result of the algorithm is very good, which also proves the effectiveness of the algorithm used in this paper.

\section{Method}

2.1. Boundary Problem of Gravity Anomaly Field. When calculating the field value of the local gravity anomaly of the earth, the curvature of the earth is not considered. In threedimensional space, the coordinate system $\mathrm{O}$-xyz can be established, which is $\mathrm{O}-\mathrm{z}$ along the vertical downward direction of gravity. At the same time, the gravitational potential caused by the abnormal body can be expressed as $V(\vec{r})$, which satisfies the Poisson equation inside, such as the formula

$$
\nabla^{2} V(\vec{r})=-4 \pi G \rho(\vec{r})
$$

where $\vec{r}$ is the vector, $G$ is the gravitational constant, and $\rho$ is the density at $\vec{r} \cdot V(\vec{r})$ satisfies the Laplace equation outside the abnormal body.

$$
\nabla^{2} V(\vec{r})=0
$$

According to formulas (1) and (2), we can obtain the gravity anomaly data any position in the space.

$$
g(\vec{r})=\frac{\partial V}{\partial x} .
$$

According to formula (3), Poisson's equation and Laplace's equation are satisfied inside and outside the abnormal body.

$$
\begin{aligned}
& \nabla^{2} g(\vec{r})=-4 \pi G \frac{\partial \rho}{\partial z}(\vec{r}), \\
& \nabla^{2} g(\vec{r})=0 .
\end{aligned}
$$

As shown in Figure 1, the area surrounded by area $A$ represents the geological body with abnormal density and $A_{0}$ represents the area without the abnormal body. When we have the abnormal value of boundary condition $S$, we can obtain the field value of any point in the space by formulas (4) and (5).

When calculating the gravity anomaly generated by the three-dimensional abnormal body, the gravity anomaly generated on $S$ can be calculated according to the density distribution law of the abnormal body. With boundary conditions, the gravity anomaly on $S$ can be calculated by Poisson equations (4) and (5). However, although the shape and density data of the abnormal body are known, the abnormal value on the closed surface $S$ is not known. Therefore, the closed surface $S$ should be selected first, and then the gravity abnormal data on the curve $S$ should be calculated [19].

2.2. Selection of Gravity Boundary Conditions. As shown in Figure 2, if the coordinate origin $o$ is selected at the center of gravity of the abnormal body $A$, the gravitational potential generated by the abnormal body $A$ at any point outside can be expressed as

$$
\begin{aligned}
V(\vec{r}) & =G \int \frac{\rho}{r_{0}} \mathrm{~d} \tau, \\
\frac{1}{r_{0}} & =\frac{1}{r} \sum_{n=0}^{\infty}\left(\frac{r^{\prime}}{r}\right)^{n} p_{n} \cos \theta .
\end{aligned}
$$

In the formula, $p_{n} \cos \theta$ is the Legendre polynomial of order $n$ of $\cos \theta$. By using the above formula, the gravity anomaly value of the abnormal body on the boundary can be obtained. After getting the gravity anomaly values on all nodes of the boundary conditions, the next step can be carried out. When the value of $r$ is larger, the data of gravity anomaly field on the boundary is more accurate, but the time of numerical simulation will be greatly increased [20]. In a comprehensive consideration, we choose the length of $r$ as about 10 times the length of the abnormal body and use the synthetic data to test the results. 


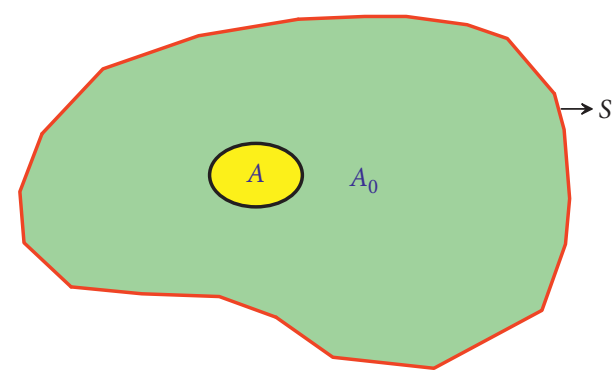

Figure 1: Diagram of the abnormal body and closed area.

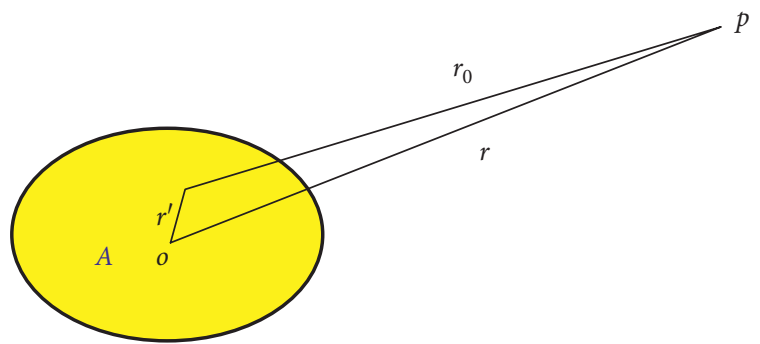

FIGURE 2: Diagram of the abnormal body and closed area.

We multiply the two ends of formula (4) by the continuous differentiable function $\varphi(\vec{r})$ and integrate them within the boundary conditions to get the following formula:

$$
\int_{A+A_{0}} \varphi \nabla^{2} g \mathrm{~d} \tau=-4 \pi G \int_{T} \varphi \frac{\partial \rho}{\partial z} \mathrm{~d} \tau .
$$

If the density of the geological body is evenly distributed along the direction of $z$ axis, then there is the following formula:

$$
\int_{T} \varphi \frac{\partial \rho}{\partial z} \mathrm{~d} \tau=-\int_{T} \rho \frac{\partial \varphi}{\partial z} \mathrm{~d} \tau
$$

Formula (9) can be obtained after simplification:

$$
\int_{T+T_{0}} \nabla \varphi \cdot \nabla g \mathrm{~d} \tau=-4 \pi G \int_{T} \rho \frac{\partial \varphi}{\partial z} \mathrm{~d} \tau .
$$

2.3. Finite Element Method of Gravity Anomaly. When we use the finite element method to calculate the gravity anomaly of the three-dimensional geological body, we need to follow these steps:

(1) According to the shape and density distribution of the abnormal body, the center of gravity of the abnormal body is calculated.

(2) Select the appropriate boundary as the boundary condition and calculate the gravity anomaly data generated by all nodes on the boundary condition.

(3) The integral equation (9) is discretized in space so that equation (9) can be transformed into a system of linear equations. The numerical solution of the Poisson equation in the whole space can be obtained by solving the system of equations.
We use tetrahedral elements to establish linear and quadratic finite element schemes. The method of the variational equation is used to obtain the gravity anomaly of grid nodes. At any point in tetrahedron $e$, the approximate solution $\varphi$ is expressed as

$$
\begin{aligned}
& g^{e}(x, y, z)=N_{i}^{e}(x, y, z) g_{i}, \\
& \varphi^{e}(x, y, z)=N_{i}^{e}(x, y, z) \varphi_{i},
\end{aligned}
$$

where $N_{i}$ is the shape function of the tetrahedral element $e$ and $i=1,2,3,4$ is the number of four vertices of tetrahedron. The shape function of tetrahedron can be expressed as the linear equation, as shown in the following equation :

$$
N_{i}^{e}=\frac{1}{6 V^{e}}\left(a_{i}^{e}+b_{i}^{e} x+c_{i}^{e} y+d_{i}^{e} z\right) .
$$

Inside the tetrahedron $e$ of the element, the left-hand side of formula (9) can be expressed as

$$
\int_{e} \nabla \varphi \cdot \nabla g \mathrm{~d} \tau=\varphi_{i} k_{i, j}^{e} g_{j},
$$

where $i$ and $j$ are the summation coordinates.

$$
k_{i, j}^{e}=\int_{e} \frac{\partial N_{i}^{e}}{\partial x_{r}} \frac{\partial N_{i}^{e}}{\partial x_{r}} \mathrm{~d} \tau,
$$

where $r$ is the summation coordinate. $k_{i, j}^{e}$ is the element of stiffness matrix $k^{e}$. The right-hand side of equation (9) can be expressed as

$$
\begin{aligned}
& -4 \pi G \int_{e} \rho \frac{\partial \varphi}{\partial z} \mathrm{~d} \tau=\varphi_{i} F_{i}^{e}, \\
& F_{i}^{e}=-4 \pi G \int_{e} \rho \frac{\partial N_{i}^{e}}{\partial z} \mathrm{~d} \tau .
\end{aligned}
$$

According to equations (15) and (16), in the tetrahedron of the element, equation (11) can be expressed as

$$
\varphi_{i} k_{i, j}^{e}=\varphi_{i} F_{i}^{e} .
$$

If $n$ is the total number of nodes in equation (9), then $k^{e}$ and $F^{e}$ can be expressed as matrices of $N \times N$ order and vectors of degree $n$, and then sum all elements to obtain the following formula:

$$
\begin{aligned}
\varphi_{i} k_{i, j} g_{j} & =\varphi_{i} F_{i}, \\
k_{i, j} & =\sum_{s} k_{i, j}^{e}, \\
F_{i} & =\sum_{s} F_{i, j}^{e} .
\end{aligned}
$$

When on the node of Dirichlet boundary condition, $\varphi_{r}=0$. Therefore, formula (19) can be obtained as follows:

$$
\varphi_{i} k_{i, j} g_{j}+\varphi_{i} k_{i, r} g_{r}=\varphi_{i} F_{i}
$$

By solving the linear equation (19), we can obtain the gravity anomaly values of all nodes in the region. 
2.4. Examples. The solution calculated by the finite element method is a numerical solution, so it needs to use the analytical solution to compare with it and the error also needs to be analyzed. When the error is within the acceptable range, the actual model can be used for calculation. In order to evaluate the algorithm used in this paper, we choose to use the rectangular abnormal body model for experiment, as shown in Figure 3 . The size of the abnormal body in the middle of Figures $3(\mathrm{a})$ and $3(\mathrm{~b})$ is $100 \times 100 \times 100 \mathrm{~m}$, the density is $1 \mathrm{~g} / \mathrm{cm}^{3}$, the density of the background is $0 \mathrm{~g} / \mathrm{cm}^{3}$, and the boundary condition is set at the position 10 times the size of the abnormal body. This boundary condition belongs to the first kind of the boundary condition, also called the Dirichlet boundary condition. According to the governing equations and boundary conditions, the gravity field of any point in the region can be calculated.

Figures 3(a) and 3(b) are rough mesh and fine mesh models, respectively. The grid quality of Figure 3(b) is relatively high and the number of grids is large, so the time of numerical simulation will be greatly increased. The number of grids in Figure 3(a) is less, and the calculation time will not be too much. The grid parameters of the two models are shown in Table 1.

As shown in Figure 4, Figure 4(a) represents the exact solution of the anomaly data $(Y=500 \mathrm{~m}$, elevation $=1000 \mathrm{~m})$. Figures $4(\mathrm{~b})$ and $4(\mathrm{~d})$ represent the numerical solutions of coarse and fine mesh $(Y=500 \mathrm{~m}$, elevation $=1000 \mathrm{~m}$ ), and Figures $4(\mathrm{c})$ and 4(e) represent the error distribution between the two and the exact solutions. From the shape of the curve, it can be seen that the error of the simulation results of the coarse mesh is large, and the error of the local position has exceeded $0.008 \mathrm{mGal}$. However, the error of the simulation results of the fine mesh is much smaller, and the error curve always fluctuates around the position of 0 point, and the maximum error is not more than $0.001 \mathrm{mGal}$. Therefore, when the grid is encrypted, it can not only effectively reduce the error of numerical simulation but also increase the running time of the computer. As shown in Table 1, the numerical simulation time of the coarse mesh is only $42.50 \mathrm{~s}$, and the numerical simulation time of the fine mesh is $1644 \mathrm{~s}$. The number of grids and the number of nodes are also increased several times.

Therefore, the above results give us some enlightenment. When the finite element method is used to calculate the gravity anomaly field of the actual geological model, it is necessary to effectively control the number of grids according to the shape of the model, so as to ensure the accuracy of the numerical simulation and save the memory of the computer as much as possible.

\section{Real Data}

3.1. Geological Background. The Jinchuan ore bearing ultrabasic intrusive body is located in the northeast of the Longshoushan uplift belt in the southwest margin, and the mining area is between $38^{\circ} \mathrm{N}$ and $39^{\circ} \mathrm{N}$. The deep fault $(F 1)$ in the north of Longshoushan uplift is adjacent to the tidal fault basin of the Alxa block, while the deep fault in the south is adjacent to the corridor transition zone of the early Paleozoic fold belt of North Qilian [21]. The Jinchuan mining area is called III, I, II, and IV mining area from west to east, as shown in Figure 5. The scale, shape, occurrence, and ore storage capacity of rock mass in each mining area are different. This paper selects the Jinchuan II mining area for research. The intrusive body of the mining area II is about $3000 \mathrm{~m}$ long, which lies in the southwest direction of fault F16. The east part is covered by a quaternary system, and the rest sections are exposed [21]. It gradually widens from east to west, with the largest width around $F 17$, about $530 \mathrm{~m}$, and then narrows to the west. The strike of the intrusion is about $50^{\circ}$ to the northwest, and the dip angle is $50^{\circ}$ to $60^{\circ}$, and the dip angle in the east is relatively slow. The west end is obviously deflected by the influence of the fault, with a large extension depth, and the maximum extension depth is more than $1000 \mathrm{~m}$, among which there is a largescale rich ore body, which is ore body 1 . The eastern part of the ore body is relatively shallow, with a wide outcrop, and the maximum width of $530 \mathrm{~m}$, which is ore body 2 . The nickel content of ore bodies 1 and 2 accounts for more than $99 \%$ of the mining area. The position of the purple line in Figure 5 represents the location of the section model in Figure 6, which is No. 34 section of Jinchuan $\mathrm{Cu}-\mathrm{Ni}$ sulfide deposit.

3.2. Modeling of Gravity. According to the drilling data, we can determine the lithologic body information of some sections. When the distance between the two sections is close, the 3D geological model can be established by the interpolation method. We first established a two-dimensional geological section model of Jinchuan $\mathrm{Cu}-\mathrm{Ni}$ sulfide deposit, which is shown in Figure 7. According to the drilling data of Jinchuan $\mathrm{Cu}-\mathrm{Ni}$ sulfide deposit, the accuracy of the cross section model can be effectively controlled. At the same time, there are other sections and drilling data in this area. Therefore, it is scientific to use the borehole data of the cross section for geological modeling. In order to better describe the boundary of rock and ore body and fine structure of rock mass and increase the number of corresponding points, the magnetic susceptibility model with uneven grid density can be obtained.

We can see the density data of each lithologic body from Figure 7 . The density of striped migmatite, serpentine marl, and homogeneous migmatite is $2.67 \mathrm{~g} / \mathrm{cm}^{3}$. Since the anomaly response calculated in this paper is residual gravity anomaly, the three lithologic bodies are taken as the background and the density data of $2.67 \mathrm{~g} / \mathrm{cm}^{3}$ is taken as the background density.

The rock mass in the green area is peridotite, and the peridotite has more components, which contributes to the largest proportion of gravity anomaly field. The density difference between peridotite and background lithologic body is $0.3 \mathrm{~g} / \mathrm{cm}^{3}$. In addition, the model also includes some high-grade ore bodies, such as intrusive sulfide ores and ultrabasic sulfide ores. The density value of them is $3.03 \mathrm{~g} / \mathrm{cm}^{3}$, and the density difference with the 


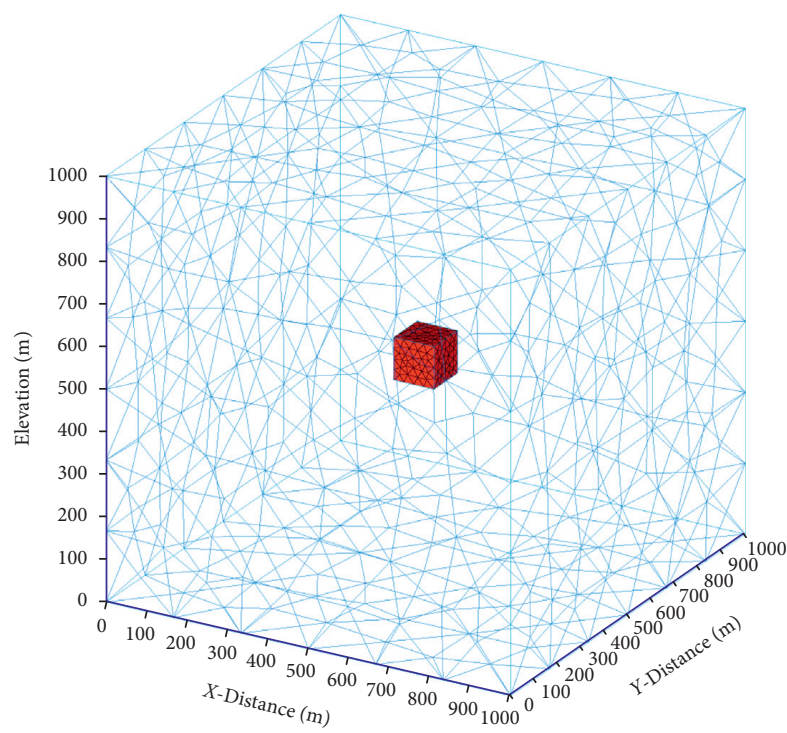

(a)

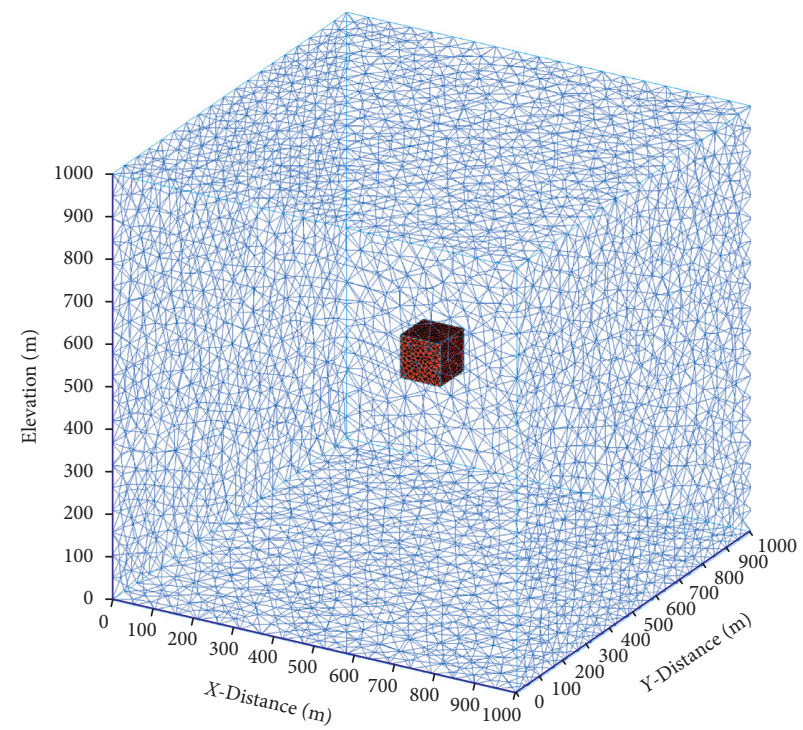

(b)

Figure 3: Synthetic data. (a) Coarse mesh. (b) Fine mesh.

TABle 1: Two model parameters after mesh generation.

\begin{tabular}{l} 
Grid/parameters \\
Number of grids \\
Average volume \\
Total point \\
Average size \\
CPU consumption time \\
\hline
\end{tabular}

(a)

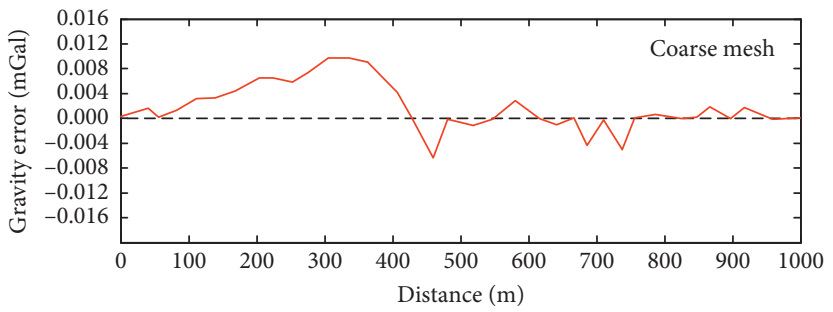

(c)

$\begin{array}{cc}\text { Fine mesh } & \text { Coarse mesh } \\ 101.821 & 22.080 \\ 8496.32 & 44251.70 \\ 22.219 & 4.331 \\ 38.093 & 57.393 \\ 1644 \mathrm{~s} & 42.50 \mathrm{~s}\end{array}$

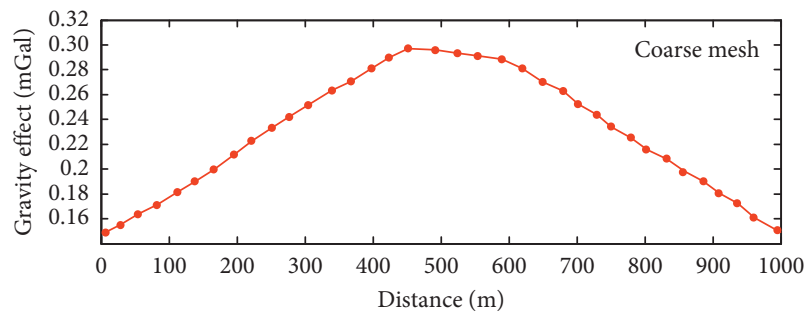

(b)

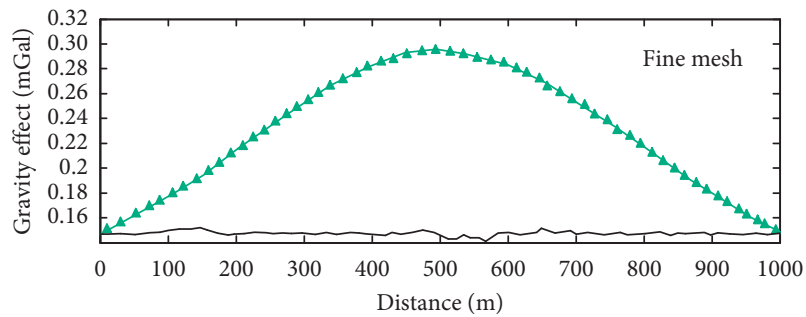

(d)

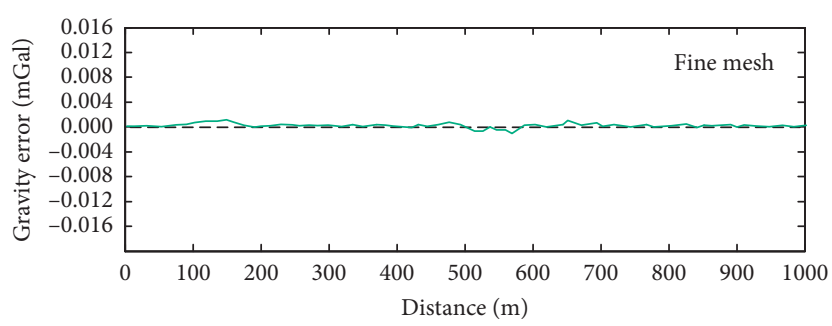

(e)

Figure 4: Calculation results of the abnormal body by different mesh generation. (a) Analytical solution. (b) Coarse mesh. (c) Difference between (a) and (b). (d) Fine mesh. (e) Difference between (a) and (d). 


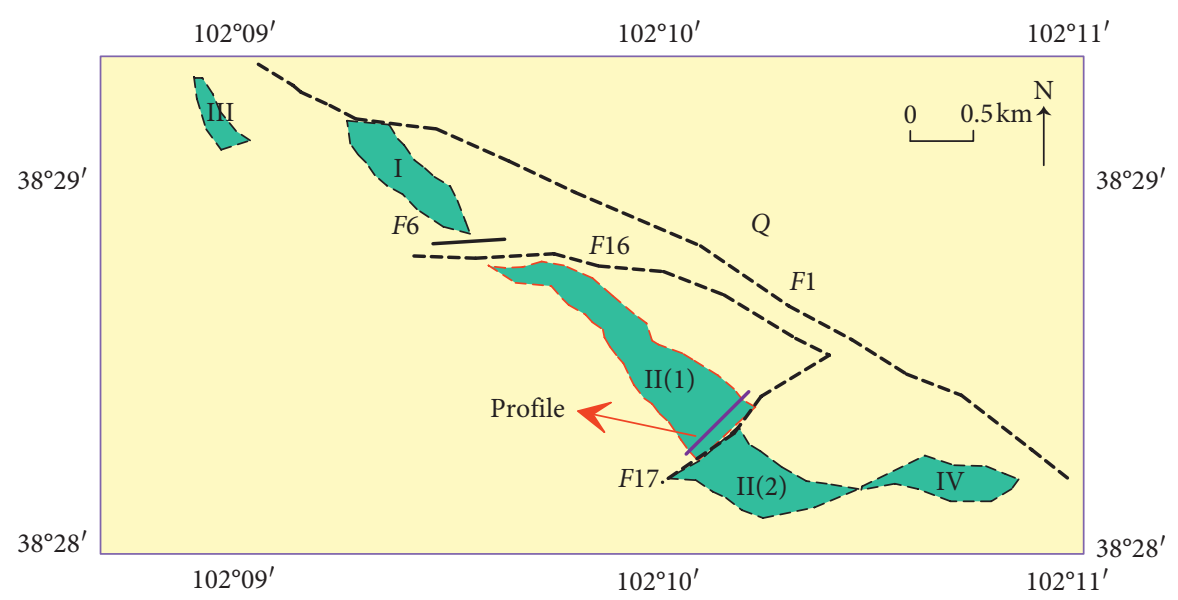

FIGURE 5: Research area.
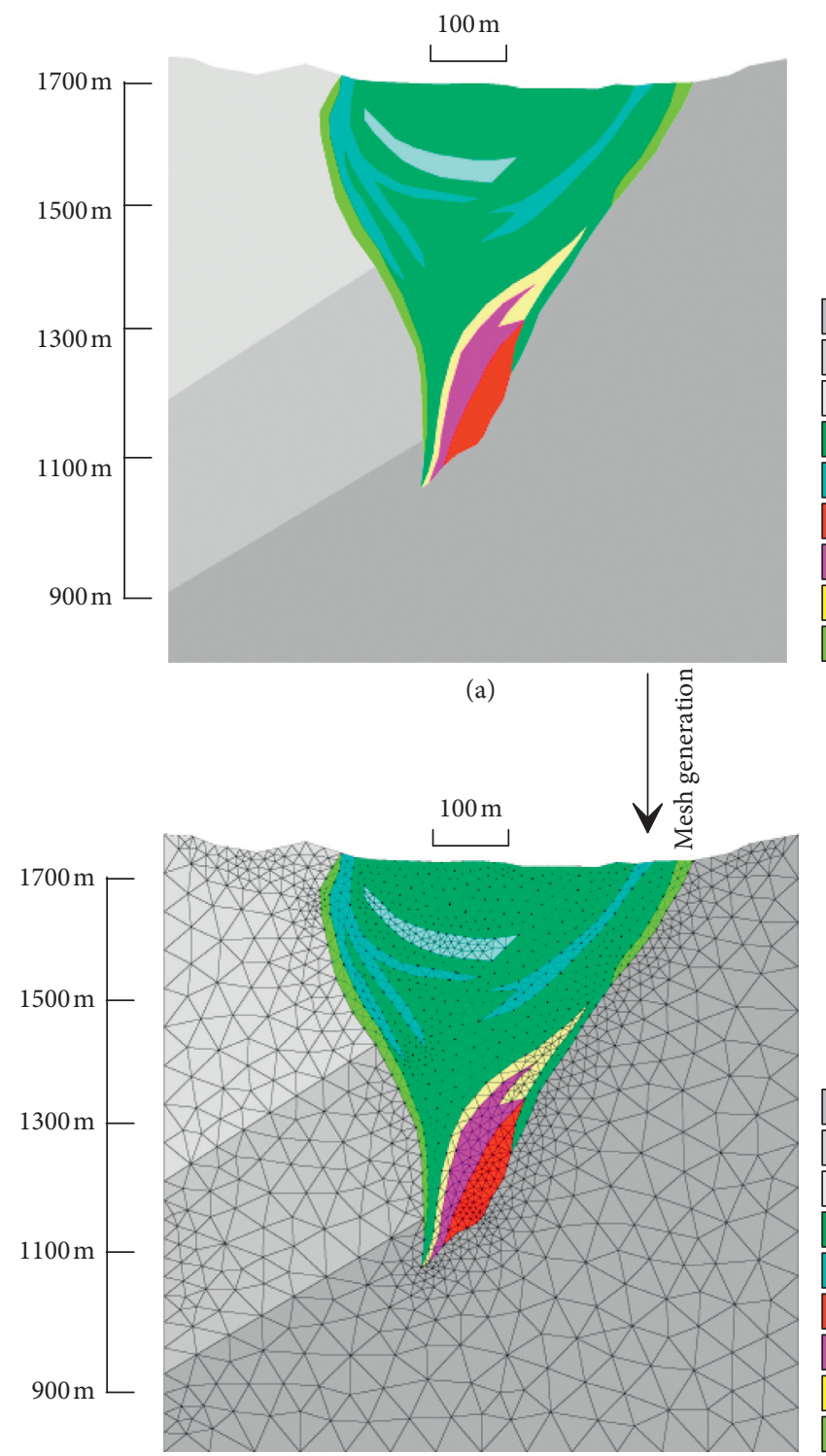

Serpentine marble $\left(2670 \mathrm{~kg} / \mathrm{m}^{3}\right)$

Striated migmatite $\left(2670 \mathrm{~kg} / \mathrm{m}^{3}\right)$

Homogeneous migmatite $\left(2670 \mathrm{~kg} / \mathrm{m}^{3}\right)$

Wollastonite $\left(2970 \mathrm{~kg} / \mathrm{m}^{3}\right)$

Anorthosite lherzolite $\left(2970 \mathrm{~kg} / \mathrm{m}^{3}\right)$

Sponge meteorite pure element $\left(3030 \mathrm{~kg} / \mathrm{m}^{3}\right)$

Disseminated sulfide pure element $\left(3030 \mathrm{~kg} / \mathrm{m}^{3}\right)$

Superbasic sulfide ore $\left(3030 \mathrm{~kg} / \mathrm{m}^{3}\right)$

Olivine $\left(2970 \mathrm{~kg} / \mathrm{m}^{3}\right)$

(b)

Serpentine marble $\left(2670 \mathrm{~kg} / \mathrm{m}^{3}\right)$

Striated migmatite $\left(2670 \mathrm{~kg} / \mathrm{m}^{3}\right)$

Homogeneous migmatite $\left(2670 \mathrm{~kg} / \mathrm{m}^{3}\right)$

Wollastonite $\left(2970 \mathrm{~kg} / \mathrm{m}^{3}\right)$

Anorthosite lherzolite $\left(2970 \mathrm{~kg} / \mathrm{m}^{3}\right)$

Sponge meteorite pure element $\left(3030 \mathrm{~kg} / \mathrm{m}^{3}\right)$

Disseminated sulfide pure element $\left(3030 \mathrm{~kg} / \mathrm{m}^{3}\right)$

Superbasic sulfide ore $\left(3030 \mathrm{~kg} / \mathrm{m}^{3}\right)$

Olivine $\left(2970 \mathrm{~kg} / \mathrm{m}^{3}\right)$

Figure 6: No. 34 section model of Jinchuan deposit. (a) Geologic model. (b) Tetrahedral model. 


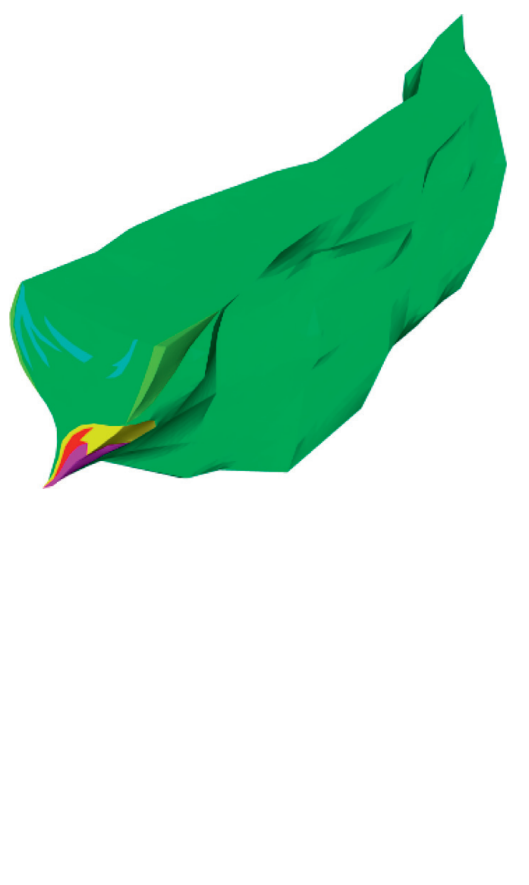

(a)

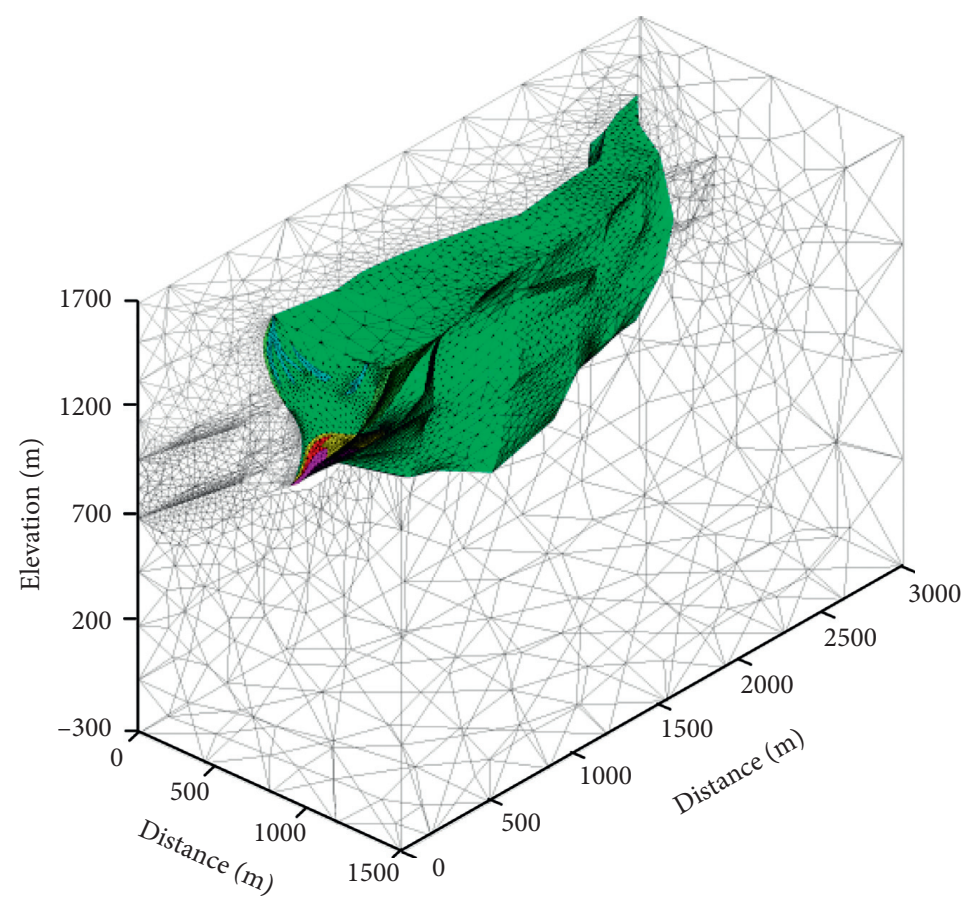

(b)

Figure 7: (a) Jinchuan deposit model. (b) Tetrahedral model.

background surrounding rock is $0.36 \mathrm{~g} / \mathrm{cm}^{3}$. With the density data of rock mass and the position information of the tetrahedral grid, the residual gravity anomaly field generated by the surface can be calculated by using the algorithm proposed in this paper. The observation system is located on the undulating surface near 1700 meters above the sea level.

The residual gravity anomaly field of the Jinchuan deposit model is calculated by the algorithm proposed in this paper, as shown in Figure 8. It can be seen that the gravity anomaly field calculated in this paper has high similarity with the Jinchuan deposit model. However, the gravity anomaly response calculated by the finite element method is only the numerical solution of the model, so we need to analyze the residual anomaly field. Therefore, this paper uses the open-source code of Growth3D to carry out 3D inversion calculation of gravity anomaly field calculated in this paper [22].

The inversion results are shown in Figure 9. It can be seen from Figure 9(a) that the inversion result is very close to the cross section model in Figure 8, and the inversion residual density value is also close to $0.3 \mathrm{~g} / \mathrm{cm}^{3}$, which corresponds well with the residual density of peridotite. At the same time, the corresponding effect of depth and width is also very good. As shown in Figure 9(c), the residual density of the surface obtained by inversion is very close to the surface deposit model, the density value is perfectly corresponding to peridotite, and the morphology has high spatial consistency with the deposit model. It can also be seen from Figure 9(b) that the maximum buried depth of the ore body obtained by inversion is about $1100-1200 \mathrm{~m}$, while the buried depth of

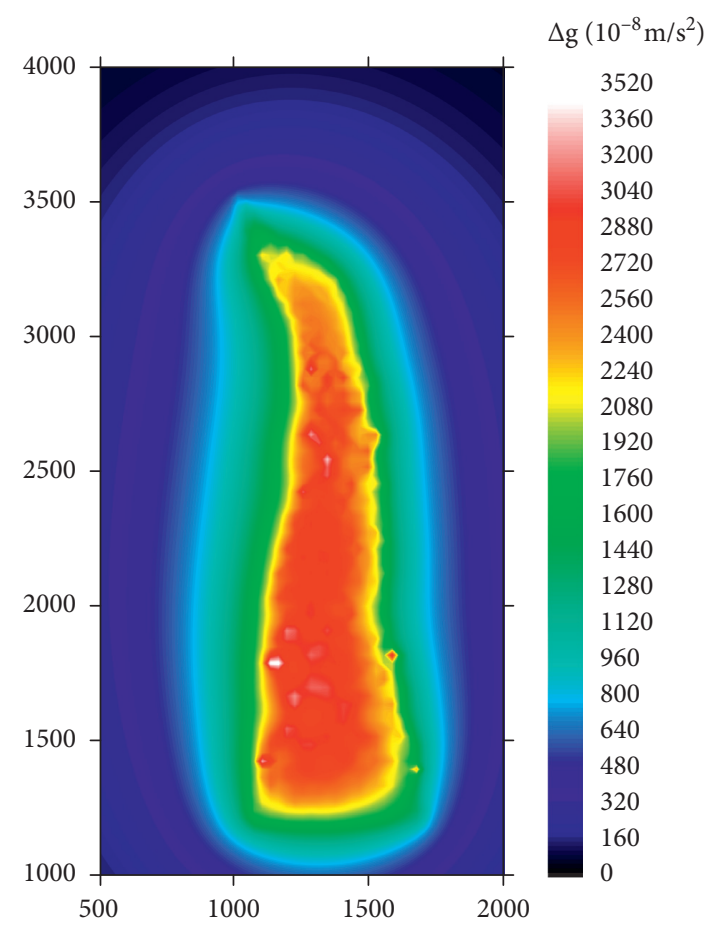

FIGURE 8: Surface gravity anomaly response.

the actual deposit is about $1100 \mathrm{~m}$. Therefore, it can be proved that the inversion results in this paper are very good. At the same time, it can be proved that the finite element method used in this paper is successful and the effect is good. 


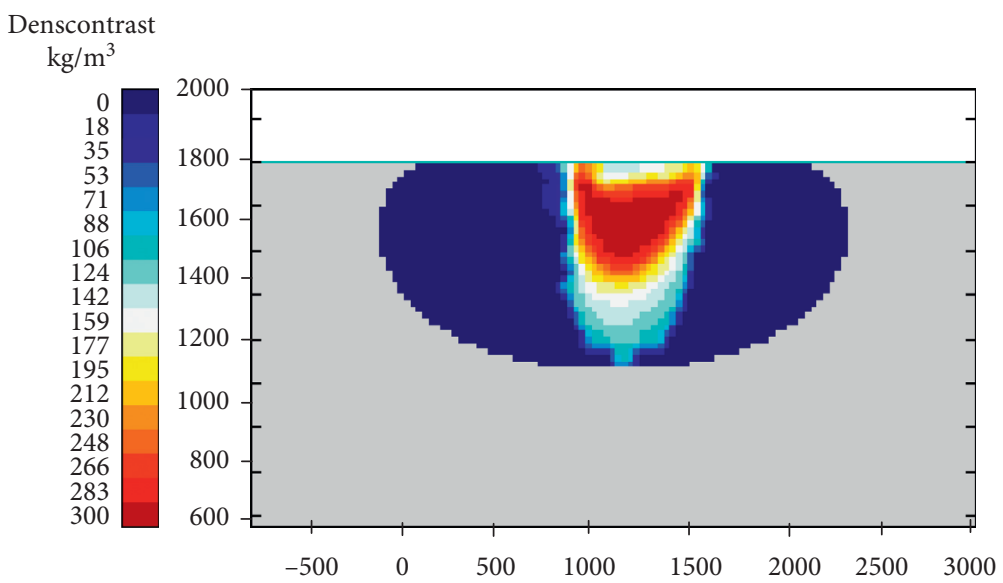

(a)

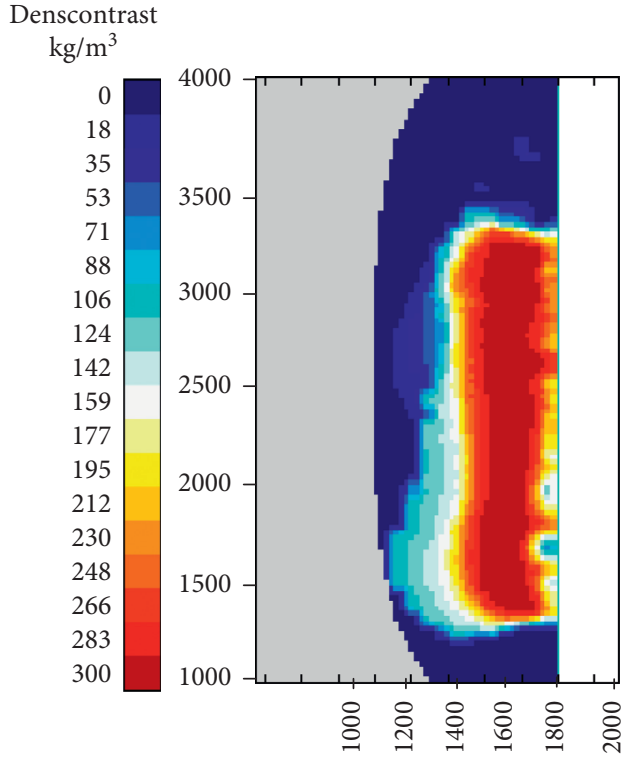

(b)

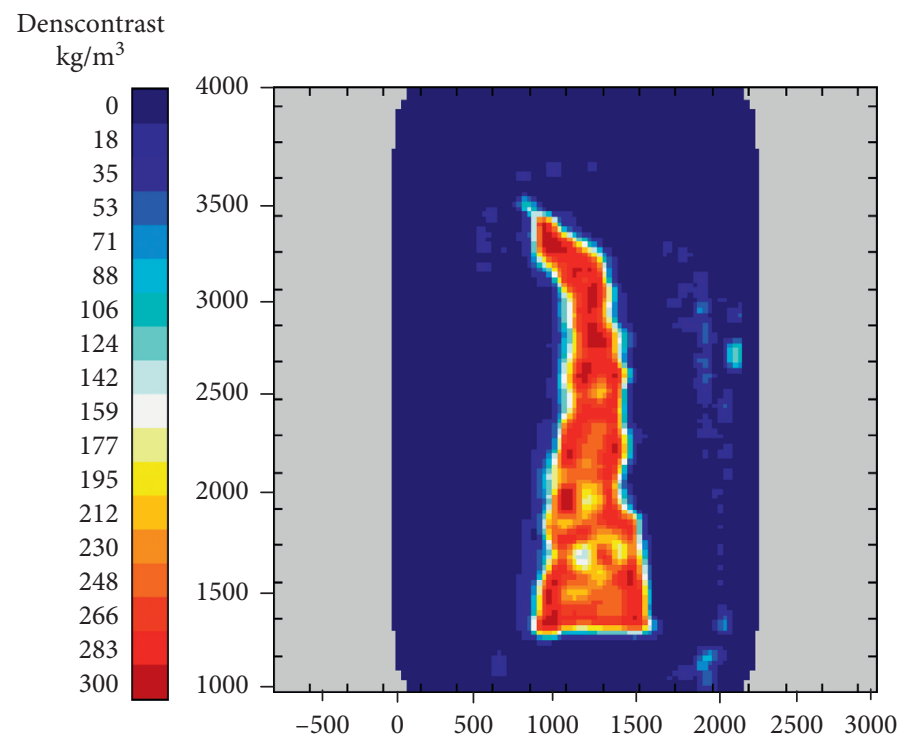

(c)

Figure 9: The inversion result of the gravity field.

\section{Conclusion}

In this paper, we use the Delaunay grid to build a complex geological model. According to the nonuniformity of density, the grid density of different regions is appropriately encrypted. Then, the finite element method is used to calculate the gravity anomaly response based on the model. First, the error analysis of the method used in this paper is carried out by using the synthetic data. Finally, the accuracy of the calculated gravity anomaly field is verified by using the inversion results, and the following three conclusions are obtained.

(1) Delaunay is a very effective method for modeling. The quality of the Delaunay grid is good, and it can complete the accurate numerical simulation. Moreover, the Delaunay grid can consider the complex structure of the model and can effectively encrypt the complex area and sparse the simple area, so as to achieve accurate modeling and save computer memory.

(2) After completing the Delaunay mesh modeling, the finite element method is used to calculate the gravity anomaly response. When the number of grids in the model is more, the accuracy of numerical simulation results is higher but the calculation time is longer. Therefore, when the number of meshes is good, the finite element method has good effect and good adaptability. 
(3) In practical application, the Delaunay mesh generation method can effectively build complex practical models. It is well performed in the modeling of Jinchuan $\mathrm{Cu}-\mathrm{Ni}$ sulfide deposit. At the same time, the results of numerical simulation are verified and analyzed by using the open-source code of Growth3D. The results show that the finite element method is a very effective method to calculate the response of gravity anomaly, which can also solve the practical engineering geological problems.

\section{Data Availability}

The data used to support the findings of this study have not been made available because the project to which the data belongs is still in progress.

\section{Conflicts of Interest}

The authors declare that they have no conflicts of interest.

\section{Acknowledgments}

The authors thank Professor Sixin Liu from College of Geoexploration Science and Technology, Jilin University, for his geologic model and useful suggestions which have greatly improved the paper. This research was supported by the National Key Research and Development Program of China (2016YFC0600505).

\section{References}

[1] R. E. White, "An introduction to the finite element method with applications to nonlinear problems," Acta Applicandae Mathematica, vol. 10, no. 3, pp. 309-312, 1987.

[2] M. Kumar and G. Mishra, "An introduction to numerical methods for the solutions of partial differential equations," Applied Mathematics, vol. 2, no. 11, pp. 1327-1338, 2011.

[3] J. M. Melenk and I. Babuška, "The partition of unity finite element method: basic theory and applications," Computer Methods in Applied Mechanics \& Engineering, vol. 139, no. 1-4, pp. 289-314, 1996.

[4] F. P. Miller, Finite Element Method in Structural Mechanics, Alphascript Publishing, Saarbrücken, Germany, 2010.

[5] C. Mei and K. Decha-Umphai, "A finite element method for nonlinear forced vibrations of rectangular plates," AIAA Journal, vol. 23, no. 7, 1985.

[6] J. R. Shewchuk, "Delaunay refinement algorithms for triangular mesh generation," Computational Geometry, vol. 22, no. 1-3, pp. 21-74, 2002.

[7] N. Amenta, B. Marshall, and M. Kamvysselis, "A new Voronoi-based surface reconstruction algorithm," International Conference on Computer Graphics and Interactive Techniques, vol. 1, no. 1, pp. 415-421, 1998.

[8] J. R. Shewchuk and B. C. Brown, "Fast segment insertion and incremental construction of constrained Delaunay triangulations," Computational Geometry, vol. 48, no. 8, pp. 554-574, 2015.

[9] K. Mallick and K. K. Sharma, "A finite element method for computation of the regional gravity anomaly," Geophysics, vol. 64, no. 2, pp. 461-469, 1999.
[10] K. K. Roy, Potential Theory in Applied Geophysics, p. 651, Springer-Verlag, Berlin, Germany, 2008.

[11] L. Zhang and T. Y. Hao, "2D irregular gravity modeling and computation of gravity based on Delaunay triangulation," Chinese Journal of Geophysics (In Chinese), vol. 49, no. 3, pp. 877-884, 2006.

[12] Z. H. Li, Z. C. Luo, and B. Zhong, "Gravity modeling and analyzing based on 3D Delaunay triangulation algorithm," Chinese Journal of Geophysics (In Chinese), vol. 55, no. 7, pp. 2259-2267, 2012.

[13] Y. Liu, Y. G. Li, and B. Han, "Delaunay edge finite element modeling of the 3d CSEM field on unstructured grids," Chinese Journal of Geophysics (In Chinese), vol. 60, no. 12, pp. 4873-4886, 2017.

[14] D. Komatitsch and J. Tromp, "Introduction to the spectral element method for three-dimensional seismic wave propagation," Geophysical Journal International, vol. 139, no. 3, pp. 806-822, 1999.

[15] J. Zhang, R. L. Mackie, and T. R. Madden, "3-D resistivity forward modeling and inversion using conjugate gradients," Geophysics, vol. 60, no. 5, pp. 1313-1325, 1995.

[16] Z. S. Jia, S. Cheng, X. Zhao, and G. Zhang, "Modeling of complex geological body and computation of geomagnetic anomaly," Mathematical Problems in Engineering, vol. 2020, no. 1, 10 pages, Article ID 9839606, 2020.

[17] A. A. Liu and I. A. Zlotnik, "A fast direct algorithm for implementing a high-order finite element method on rectangles as applied to boundary value problems for the Poisson equation," Doklady Mathematics, vol. 95, no. 2, pp. 129-135, 2017.

[18] Y. Li and D. W. Oldenburg, "3-D inversion of gravity data," Geophysics, vol. 63, no. 1, pp. 109-119, 1998.

[19] H. Jahandari and C. Farquharson, "Forward modeling of gravity data using finite-volume and finite-element methods on unstructured grids," Geophysics, vol. 78, no. 3, 2013.

[20] S. Xu, Finite Element Method in Geophysics, Science Press, Beijing, China, 1994.

[21] X. Song, "Siderophile and chalcophile elemental constraints on the origin of the Jinchuan Ni-Cu-(PGE) sulfide deposit, NW China," Geochimica et Cosmochimica Acta, vol. 73, no. 2, pp. 404-424, 2013.

[22] A. G. Camacho, F. G. Montesinos, and R. Vieira, “A 3-D gravity inversion tool based on exploration of model possibilities," Computers \& Geosciences, vol. 28, no. 2, pp. 191-204, 2002. 